\title{
Analysis of the engine test cycles from China VI heavy duty vehicle standard and China automotive test cycle
}

\author{
Xiaowei Wang ${ }^{1, *}$, Chuanqi Wang ${ }^{1,2}$, Tao Gao ${ }^{1}$, Tengteng $\mathrm{Li}^{1,2}$, and Hailiang Lao ${ }^{1}$ \\ ${ }^{1}$ CATARC Automotive Test Center (Tianjin) Co., Ltd., Tianjin 300300, China \\ ${ }^{2}$ School of Mechanical Engineering, Tianjin University, Tianjin 300072, China
}

Keywords: diesel engine, China VI standard, China automotive test cycle, regression analysis, emissions.

\begin{abstract}
This paper studied the engine test cycles including world harmonized steady cycle (WHSC), world harmonized transient cycle (WHTC), china heavy-duty steady cycle (CHSC) and china heavy-duty transient cycle (CHTC) based on a diesel engine which meet the China VI heavy duty vehicle emission standard. The results show that regression analysis of speed, torque and power all meet the requirements of the China VI heavy duty vehicle standard. For this engine, NOx, PM and THC pollutants under CHSC are $134.5 \%, 29.6 \%$ and $94.4 \%$ higher than those under WHSC, respectively. PN emissions of CHSC is $65.6 \%$ lower than that of WHSC. NOx, PM and PN pollutants under CHTC are $62.9 \%$, $96.4 \%$ and $64.3 \%$ higher than those under WHTC, respectively. The exhaust temperature of the first 350 seconds at CHTC is lower, which poses a greater challenge to the conversion efficiency of the after-treatment system at low speed and low load.
\end{abstract}

\section{Introduction}

The fast-growing Chinese automobile industry has become one of the pillars of China's economy. However, with the rapid increase in car ownership, the environmental problems have also attracted more and more attention. The exhaust pollution of diesel vehicles, especially heavy-duty diesel vehicles, is even more serious. In the "China Motor Vehicle Environmental Management Annual Report (2018)", diesel trucks only accounts for $7.8 \%$ of automotive ownership, emitting $57.3 \%$ of nitrogen oxides and $77.8 \%$ of particulate matter [1].

The GB 17691-2018 "Limits and measurement methods for emissions from diesel fuelled heavy-duty vehicles" (China VI) issued in 2018 adopts the world harmonised transient cycle (WHTC) and the world harmonised steady state cycle (WHSC) for type certification [2]. In the development process of the above operating conditions, the actual driving data were mostly from European and American countries rather than China. Actually, there is a certain driving deviation between China and other countries. In this

\footnotetext{
*Corresponding author: wangxiaowei@catarc.ac.cn
} 
context, China Automotive Technology Research Center Co., Ltd. took the lead in the development of the "China Automotive Driving Conditions" National Standard Part 3: Engines, which has now entered the stage of soliciting opinions [3]. The engine operating conditions are also composed of transient cycle (CHTC) and steady state operating cycle (CHSC).

In order to study the cycle difference between GB 17691-2018 standard and China automotive driving conditions of heavy-duty vehicles, this paper runs the above steady state and transient cycles on a diesel engine, then compares and analyzes emission differences under above cycles.

\section{Experimental setup}

\subsection{Engine and equipment}

This paper selects a $2.8 \mathrm{~L}$ diesel engine that meets the China VI emission standard. The emission control technical route is diesel oxidation catalyst (DOC) + diesel particle filter $(\mathrm{DPF})+$ selective catalytic reduction catalyst $(\mathrm{SCR})+$ ammonia catalyst (ASC). The main technical parameters of the test diesel engine are shown in Table 1.

Table 1. Main parameters of tested engine.

\begin{tabular}{cc}
\hline Parameter & Value \\
Engine capacity & $2.78 \mathrm{~L}$ \\
Bore $\times$ Stroke & $94 \mathrm{~mm} \times 100 \mathrm{~mm}$ \\
Rated power & $115 \mathrm{~kW}$ \\
Maximum Torque & $450 \mathrm{Nm}$ \\
Technical route & DOC $+\mathrm{DPF}+\mathrm{SCR}+\mathrm{ASC}$ \\
Emission Level & China VI \\
\hline
\end{tabular}

The main equipment used in this paper is shown in Table 2

Table 2. Test equipment.

\begin{tabular}{cc}
\hline Equipment name & Equipment Type and Manufacturer \\
\hline AC Dynamometer & AVL INDY P44 \\
Test bed control system & AVL PUMA Open V1.5.3 \\
Intake air temperature & conditioning \\
Gaseous emission & AVL Air Conditioning System 1600 \\
measurement & AVL Emission Bench AMA i60 \\
Particle number (PN) & AVL 489 \\
Fuel consumption & measurement \\
Particle sampling & AVL 753C/735S \\
Balance & AVL SPC 472 \\
\end{tabular}

\subsection{Test procedure}


According to the requirements of GB 17691-2018 standard, WHTC in hot and cold state, WHSC in hot state, and CHTC in cold and hot state and CHSC in hot state were run respectively. The calculation method of emissions completely followed the method provided by GB 17691-2018 standard.

\section{Results and analysis}

\subsection{Comparison of CHSC and WHSC}

Figure 1 shows the comparison of CHSC and WHSC. Both cycles include 13 operating points. The size of the bubble of each operating point is proportional to run time in the total cycle. It can be seen from Figure 1 that the speed and load assessed by CHSC are lower. The maximum normalized speed of CHSC is $60 \%$, while WHSC is $75 \%$. The lowest normalized load for CHSC except idling conditions is $10 \%$, while WHSC is $25 \%$.

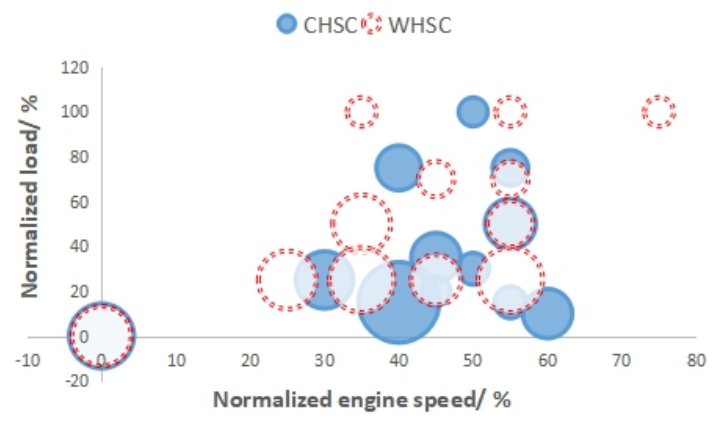

Fig. 1. Operating points comparison of CHSC and WHSC.

The regression analysis of CHSC is shown in Figures 2-4. As can be seen from the figures, the slopes of the regression lines for speed, torque, and power are 1.002, 0.9981, and 0.9986, the correlation coefficients are 1, 0.9988, and 0.9993; the intercepts are 0.3761, 0.322 , and 0.0523 , respectively. In addition, the standard deviations (SEE) of the regression lines for speed, torque, and power are $0.4,3.46$, and 0.53 respectively, which all meet the tolerance of the WHSC regression line in Table C.3 of GB 17691-2018 standard. The results indicate that the CHSC of this engine can be well followed.

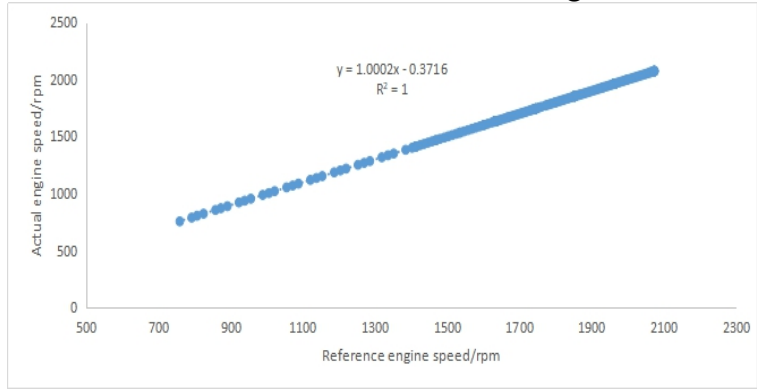

Fig. 2. Engine speed regression analysis of CHSC. 

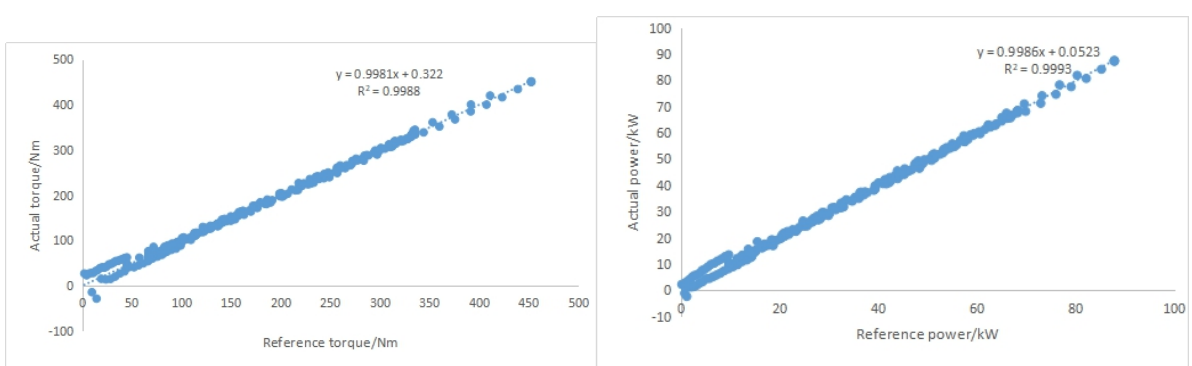

Fig. 3. Engine torque regression analysis of CHSC. Fig. 4. Engine power regression analysis of CHSC.

Run WHSC and CHSC sequentially on the selected engine, and the comparison of related operating data and emission results is shown in Table 3. It can be seen that the total duration of CHSC and the idle duration are lower than WHSC,. The total power of CHSC is $22.02 \mathrm{~kW}$, which is $17.5 \%$ lower than that of WHSC. CHSC is more biased towards low-load conditions.

Emissions for both CHSC and WHSC can meet the requirements of GB 17691-2018 standard. NOx, PM and THC pollutants of CHSC are 134.5\%, 29.6\% and 94.4\% higher than WHSC respectively. For PN emissions, CHSC is $65.6 \%$ lower than WHSC. CO emissions for both CHSC and WHSC are below the detection limit. Since the overall speed and load of CHSC are lower than WHSC, resulting in a lower exhaust temperature which causes an increase in NOx emission for CHSC. At the same time, incomplete combustion will cause an increase in THC and CO2 emissions. the load of CHTC is low so the PN emissions are reduced. The increase in PM emission may be due to incomplete combustion leading to an increase in volatile organic compounds [4].

Table 3. Comparison of CHSC and WHSC.

\begin{tabular}{cccc}
\hline Parameter & CHSC & WHSC & Difference/\% \\
\hline Total time/s & 1800 & 1895 & -5.01 \\
Idle time/s & 360 & 420 & -14.29 \\
Idle percentage/\% & 20 & 22 & -9.09 \\
Total power/kW & 22.02 & 26.7 & -17.53 \\
NOx/g/kW.h & 0.2643 & 0.1127 & 134.52 \\
PN/\#/kW.h & $6.47 \mathrm{E}+10$ & $1.88 \mathrm{E}+11$ & -65.59 \\
PM/g/kW.h & 0.0035 & 0.0027 & 29.63 \\
CO/g/kW.h & 0 & 0 & 0 \\
CO2/g/kW.h & 779.28 & 748.73 & 4.08 \\
THC/g/kW.h & 0.0241 & 0.0124 & 94.35 \\
\hline
\end{tabular}

\subsection{Comparison of CHTC and WHTC}

The operating conditions comparison of CHTC and WHTC is shown in Figures 5-7. The total time of CHTC and WHTC are 1800 seconds. For this engine, the average engine speed of CHTC is $1483 \mathrm{rpm}$, while the average engine speed of WHTC is $1568 \mathrm{rpm}$. It can be seen from Figure 7 that the medium and low speed operating condition of CHTC is more 
than that of WHTC, while the high-speed operating condition of CHTC is less than that of WHTC.

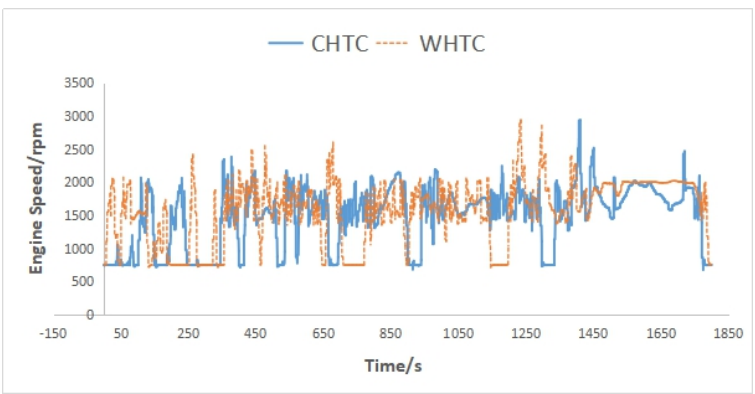

Fig. 5. Engine speed comparison between CHTC and WHTC.

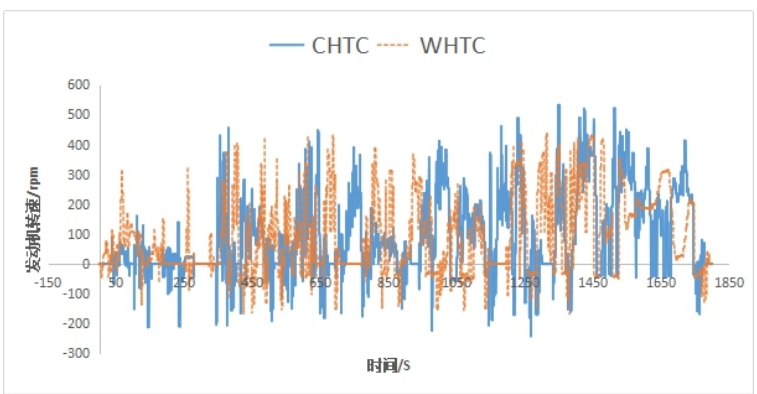

Fig. 6. Engine torque comparison between CHTC and WHTC.

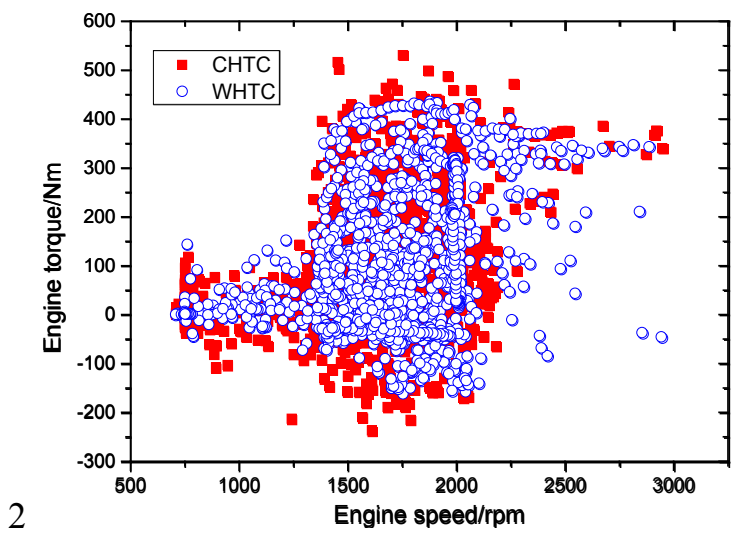

Fig. 7. Engine operating points distribution of CHTC and WHTC.

The regression analysis of CHTC is shown in Figures 8-10. As can be seen from the figures, the slopes of the regression lines for speed, torque, and power are $0.9995,0.9798$, and 0.976 , the correlation coefficients are $0.9999,0.9036$, and 0.9219 , the intercepts are $0.7561,2.6089$, and 0.5533 respectively. In addition, the standard deviations (SEE) of the regression lines for speed, torque, and power are 3.47,36.49, and 6.63 respectively, which all meet the tolerance of the WHTC regression line in Table C.2 of GB 17691-2018 standard. The results indicate that the CHTC of this engine can be well followed. 

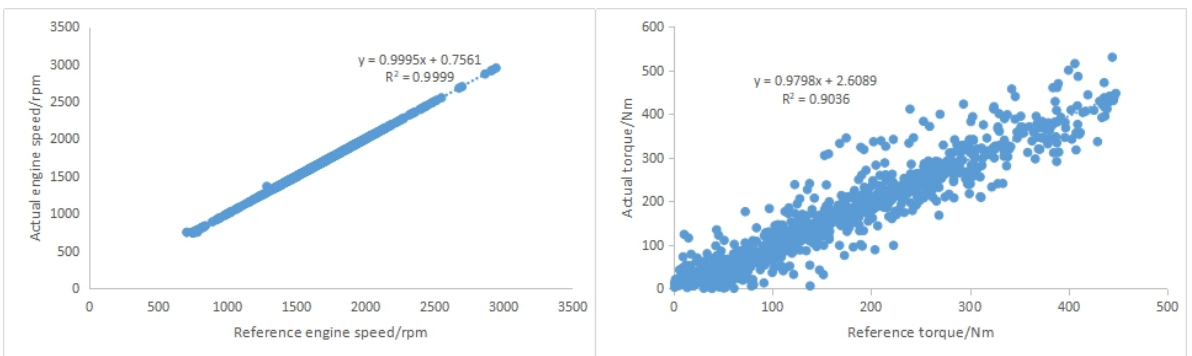

Fig. 8. Engine speed regression analysis of CHTC. Fig. 9. Engine torque regression analysis of CHTC.

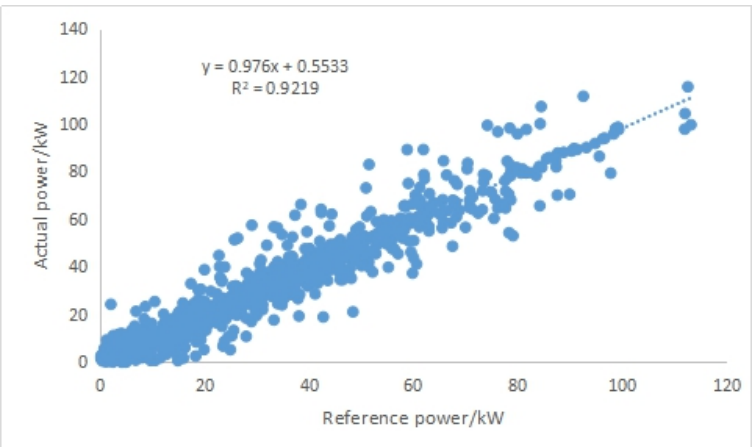

Fig. 10. Engine power regression analysis of CHTC.

Run WHTC and CHTC sequentially on the selected engine, and the comparison of related operating data and emission results is shown in Table 4.. The total power of CHTC is $18.75 \mathrm{~kW}$, which is about $4 \%$ lower than that of WHTC. Emissions for both CHTC and WHTC can meet the requirements of GB 17691-2018 standard. However, the various pollutants emissions of CHTC are higher than those of WHTC. NOx, PM and PN pollutants of CHTC are increased by $62.9 \%, 96.4 \%$ and $64.3 \%$ respectively compared with WHTC. This results show that enterprises need to make targeted calibrations focusing on CHTC.

Table 4 Comparison of CHTC and WHTC(cold plus hot state)

\begin{tabular}{cccc}
\hline Parameter & CHTC & WHTC & Difference/\% \\
\hline Total time/s & 1800 & 1800 & 0 \\
Total power/kW & 18.60 & 19.53 & -4.76 \\
NOx/g/kW.h & 0.2663 & 0.1635 & 62.87 \\
PN/\#/kW.h & $9.31 \mathrm{E}+10$ & $4.74 \mathrm{E}+10$ & 96.41 \\
PM/g/kW.h & 0.0046 & 0.0028 & 64.29 \\
CO/g/kW.h & 0.0375 & 0.0261 & 43.68 \\
CO2/g/kW.h & 783.5 & 774.23 & 1.2 \\
THC/g/kW.h & 0.0183 & 0.0056 & 226.79 \\
\hline
\end{tabular}

Figures 11 and 12 show the exhaust temperature and transient NOx emissions variation of WHTC and CHTC in the cold and hot states. It can be seen that the exhaust temperature of CHTC is significantly lower than that of WHTC in the first 350 seconds, regardless of cold or hot state. This is mainly because the speed and torque of CHTC in the first 350 seconds are relatively low. From the results of the transient NOx emission, the NOx 
emission of CHTC in the first 350 seconds is significantly higher than that of WHTC, which brings challenges in catalytic conversion efficiency under low exhaust temperature.

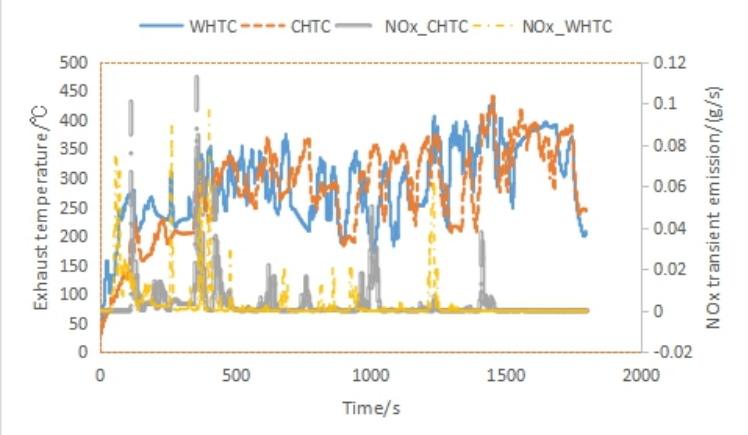

Fig. 11. Exhaust temperature and NOx transient emission of cold CHTC and WHTC.

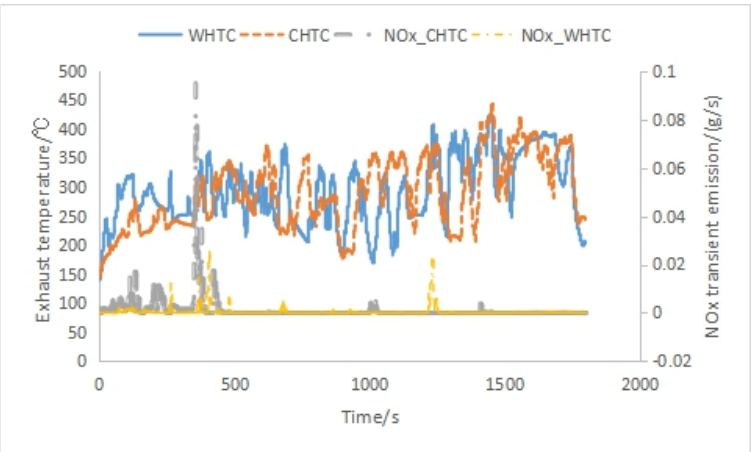

Fig. 12. Exhaust temperature and NOx transient emission of hot CHTC and WHTC.

\section{Conclusions}

By running the steady state cycles (CHSC, WHSC) and the transient cycles (CHTC,WHTC) of a diesel engine which meets the GB 17691-2018 heavy duty engine emission requirements, it is found that:

(1) The regression analysis results of speed, torque and power of both CHSC and CHTC all meet the requirements of GB 17691-2018 standard, indicating that the new steady-state and transient-state cycles can be followed very well.

(2) For this engine whose calibration based on WHSC and WHTC, almost all emissions of CHSC and CHTC are higher than WHSC and WHTC.

(3) The low exhaust temperature in the first 350 seconds of CHTC will pose a greater challenge to the conversion efficiency of after-treatment system.

This research was financially supported by CATARC Project (TJKY1920020).

\section{References}

1. China Mobile Source Environmental Management Annual Report [R]. Ministry of Ecology and Environment 2019. 
2. GB17691-2018 Limits and measurement methods for emissions from diesel fueled heavy-duty vehicle (Chinese VI) [S]. Ministry of Ecology and Environment 2018.

3. GB/T 38146.3-XXXX China automotive test cycle-Part III: Engine [S], Draft for comments. 2020.

4. Liu Xunjun. Emissions and control of internal combustion engines [M]. Beijing: Machinery Industry Press, 2005. 